Gynäkol Rundsch 1989;29(suppl. 2):I-XVI

\title{
Contents, Vol. 29, Supplement 2, 1989
}

\section{Inhalt IX}

Schröcksnadel, H.; Heim, K.: Präoperative Markierung nicht palpabler Mammabefunde mit dem Lochrastertubus 228

Untch, M.; Harbeck, N.; Eiermann, W.: Mikrometastasen im Knochenmark zum Zeitpunkt der Primärtherapie beim Mammakarzinom: Ein neues Prognosekriterium? 231

Ronay, G.; Tomesch, A.; Tulusan, A. H.: Mikrometastasen in axillären Lymphknoten beim Mammacarcinom 234

Gerstner, G. J.; Tuchmann, A.; Friedl, H. P.: Epidemiologie des Mammakarzinoms in Österreich 1983-1987 236

5. Hauptthema: Pränatale Diagnostik

Hauptreferat

Gloning, K.-Ph.; Brusis, E.: Invasive pränatale Diagnostik: Methoden, Perspektiven, Grenzen 241

Koreferat

Huber, J. C.: Pränataldiagnostik

Mitteilungen zum 5. Hauptthema

Nowotny, Christine; Endler, Margit; Derfler, K.; Feiks, A.; Lapin, A.; SunderPlassmann, G.; Balcke, P.: Die praenatale Diagnostik aus internistischer Sicht 251

Häusler, M.; Hofmann, H. M. H.; Hofer, P.; Schaffer, M.; Ipsiroglu, O.:

Steirische Erhebung angeborener Fehlbildungen mit regionaler Zuordnung und internationalen Vergleichswerten , .254

Brusis, E.; Gloning, K. Ph.; Schramm, Th.; Ostermayer, E.; Kuss, E.: Verbesserte intrauterine Diagnostik und Therapie bei Blutgruppenunverträglichkeit 256

Abet, L.; Natho, W.; Bollmann, R.; Prenzlau, P.; Bartho, S.: Wert der Amniofetographie (AFG) für die pränatale Diagnostik 260

Ostermayer, Eva; Gloning, K. Ph.; Schramm, Th.; Brusis, E.; Kuss, E.: Präpartale Punktion fetaler Ovarialzysten - eine Alternative zur Ovarektomie bei Neugeborenen? 265

Wisser, J.; Nerlich, A.; Schweitzer, B.; Krone, S.; Knitza, R.: Fetale Zwergwuchsformen - Versuch einer antenatalen Differentialdiagnostik 268 Schramm, Th.; Gloning, K.-Ph.; Brusis, E.; Ostermayer, Eva: Pränatale Diagno stik und Therapie thorakaler Entwicklungsstörungen 270

Krone, S.; Wisser, J.; Uberfuhr, J.; Angerpointner, T.; Versmold, H.: Pränatale Diagnostik der angeborenen Zwerchfellhernie $\quad 272$

Rempen, A.: Pränatale Diagnostik von Defekten der Bauchhöhlenwand 274 Knitza, R.; Hasbargen, U.; Ott, M.; Wisser, J.; Hepp, H.: Stellenwert der 
pränatalen Gewichtsschätzung bei höhergradigen Mehrlingen

Inhalt

Editorial $\quad \mathrm{XV}$

Dapunt, O.; Holzmann, K.: Grußwort 1

Fikentscher, R.: Tradition als lebendige Verpflichtung 5

I. Hauptthema: Die Wertigkeit des vaginalen operativen Eingriffs

Hauptreferat Dapunt, O.: Die Wertigkeit des vaginalen Vorgehens bei der Harninkontinenz . 11

Koreferate

Schüßler, B.: Operatives Vorgehen bei der Kombination aus Descensus vaginalis

und Streßinkontinenz 19

Ralph, G.; Winter, R.; Lang, P.: Die Bedeutung der Diaphragmaplastik in der

Therapie der Streßinkontinenz 25

Kölbl, H.: Die Korrektur der Beckenbodeninsuffizienz - Eine Domäne des

vaginalen Operationsweges 30

Mitteilungen zum 1. Hauptthema

Winter, R.; Ralph, G.: Die Vaginaefixatio sacrospinalis vaginalis als Behandlung

des prolabierten Scheidenblindsackes

34

Vytiska-Binstorfer, Elisabeth; Huber, J. C; Gitsch, E.; Kurz, Ch.; Schultz, A.:

Belastungsparameter bei vaginaler und abdominaler Hysterektomie 36

Albrich, W.: Die vaginale sacrospinale Fixation der Vagina zur Behebung des

echten Scheidenblindsackvorfalles 40

Schwarz, R.; Gerber, B.: Erfahrungen mit der vaginalen Hysterektomie 43

Gitsch, G.; Berger, Eva; Tatra, G.: 30 Jahre vaginale Hysterektomie an der

II. Universitäts-Frauenklinik Wien, eine Analyse von über 6000 Operatio-

nen 45

Gründling, H.; Golob, E.; Schausberger, L.: 2121 vaginale Uterusexstirpationen

1979-1988 47

Inhalt

VI

Zieger, W.; Hettenbach, A.; Wischnik, A.; Melchert, F.: Zur Indikationsstellung von vaginalen Hysterektomien nach vorangegangener gynäkologischer Laparotomie 52

Stummvoll, W.; Nagl, F.; Prestel, A.; Wallner, J.: Zur Hysterektomie: 1st der vaginale Weg sicherer als der abdominale? 54

Lembke, J.; Göretzlehner, G.; Nikschick, Sabine: Nachbehandlung der Kolpo-

poesis peritonei 57

Anthuber, C; Obernitz, N. v.; Denecke, H.: Stool-Outlet-Obstruction bei

Rekto-Enterozele. Inzidenz, Diagnostik und postoperative Ergebnisse .... 59

2. Hauptthema: HPV-Infektion und Zervixkarzinom

Hauptreferat Girardi, F.; Fuchs, P.; Haas, J.: Die klinische Bedeutung der HPV-Infektion ... 61

Koreferat

Breuel, C.; DiPaolo, M.; Lang, N.: Der Stellenwert der Portioabschabung bei

Dysplasien der Portio 67

Mitteilungen zum 2. Hauptthema 
Müller-Holzner, Elisabeth; Holböck, Elisabeth; Sachsenmaier, M.; Dapunt, O.: Untersuchungen über die Zunahme und Wertigkeit des zytologischen Befundes PAP III D 73

Holböck, Elisabeth; Müller-Holzner, Elisabeth; Sachsenmaier, M.; Dapunt, O.: Klinische und histologische Befunde bei peristierendem PAP III D 75 Wartusch, Barbara; Müller-Holzner, Elisabeth; Weiss, D,; Heim, K.; Holböck, Elisabeth; Fuith, L.: Inzidenz der Papillomavirustypen 6/11 und 16/18 in Zervikalabstrichen 79

Czerwenka, K. F.; Schön, H. J.; Manavi, M.; Knogler, W.: HumanePapillomavirus-Infektion als onkogener Faktor bei der Pathogenese des Zervixkarzinoms 81

Rudolf, K.; Barten, Gunhild; Wilken, H.: Verlauf papillomvirusbedingter Veränderungen im Zervixbereich während der Gravidität 85

Mayer, H. O.; Girardi, F.; Pickel, H: Mikrobiologische Befunde einschließlich Chlamydienserologie bei zervikaler Dysplasie und HPV-Infektion 88

Schön, H. J.; Czerwenka, K. F.; Manavi, M.; Knogler, K.: Die Bedeutung bakterieller und viraler Mehrfachinfektionen bei der Genese zervikaler Dysplasien 91

Reichel, R.; Fitz, R.; Budiman, R.; Czerwenka, K.; Breitenecker, G.; Denison, Ursula: Die Behandlung der virusassoziierten Zervixdysplasie mit rekombinantem Interferon alpha-2c oder gamma 94 Heim, K.; Krause, P.; Huter, O.; Wartusch, B.; Holböck, Elisabeth; Conrad, F.; Bichler, A.; Müller-Holzner, Elisabeth: Lasertherapie von Kondylomen im weiblichen Genitalbereich 96

Friedmann, W.; Schäfer, A.; Lichtenegger, W.: Zytologische Zeichen der HPVInfektion bei HIV-positiven Patientinnen 100

Inhalt

VII

Ortner, A.: Analyse der PAP-III-D-Befunde als Diskussionsgrundlage für klinische Konsequenz 103

3. Hauptthema: Der vorzeitige Blasensprung - Infektion - Tokolyse Hauptreferat

Wulf, K. H.: Der vorzeitige Blasensprung aus klinischer Sicht 106 Koreferate

Martius, J.: Vorzeitiger Blasensprung und vaginale Infektionen

Ludwig, H.: Eihaut und Dezidua - Morphologie und biomolekulare Wege ihrer Aktivierung am Beispiel des vorzeitigen Blasensprungs

Lechner, W.: Neue Entwicklung in der Tokolyse 122

Grospietsch, G.; Boenisch, H.: Vorzeitiger Blasensprung und Tokolyse?

Salzer, H.: Vorzeitiger Blasensprung und fetale Lungenreife

Rudelstorfer, R.; Gruber, W.; Janisch, H.: Vorzeitiger Blasensprung bei drohender Frühgeburt - „Fetal outcome” bei abwartendem Management

Husslein, P.: Die Rolle der Prostaglandine beim vorzeitigen Blasensprung

Kosian, K.: Die amniale Infektion beim vorzeitigen Blasensprung

Mitteilungen zum 3. Hauptthema 
Welsch, H.; Krone, H. A.: Zur Letalität der Mutter beim vorzeitigen Blasen sprung 150

Mayer, H. O.; Auner, J.: Bakteriologische Befunde bei vorzeitigem Blasen sprung 152

Renziehausen, K.; Müller, K.; Tiller, R.; Förster, St.: Über den Einfluß des vorzeitigen Blasensprunges auf die mütterliche und kindliche Morbidität (Analyse an 219 hochgradig unreifen Frühgeborenen mit einem Gewicht von 1000-1499 g) 155

Karpf, E. F.; Mayer, H. O.: Die histologische Diagnose der Amnioninfektion an der Placenta 158

Wunderer, G.; Röhrig, E.; Suschke, H.; Walter, I.: Leukozyten-Elastase als Indikator zur Früherkennung der Neugeboreneninfektion 161

Bichler, A.; Kainhofer, A.; Bayr, M.: Nosokomiale puerperale Infektionen nach Vaginalgeburten: Pathogenese, Prophylaxe 163

Mursch-Edlmayr, G.; Hintermüller, P.; Bohaumilitzky, Th.; Fröhlich, H.: Geburtshilfliche Management beim vorzeitigen Blasensprung an der LFK Linz .... 166 Hümpfner, A.; Heidegger, H.: Beta-2-Mikroglobulinurie und Lysozymurie: Bedeutung als diagnostischer und therapeutischer Kontrollparameter bei Dila tation der oberen Harnwege in der Schwangerschaft 169 Chalubinski, Kinga; Endler, Margit; Gruber, W.: Behandlung der Pyelitis gravidarum mit Pivmecillinam 171

Schön, H. J.; Czerwenka, K. F,; Manavi, M.; Linhardt, L.: Chlamydia trachomatis-Infektion in der Schwangerschaft 173

Inhalt

VIII

Schlögl, H.; Rudelstorfer, R.; Kosian, K.; Bieglmayer, Ch.: Chlamydieninfektion in der Schwangerschaft: Screening von Müttern und deren Neugeborenen . . 177 Gloning, K.-Ph.; Schramm, Th.; Brusis, E.; Schwarz, T.; Roggendorf, M.: Nonimmun Hydrops Fetalis durch Parvo-Virus-Infektion: Diagnostik und erfolgreiche Behandlung $\quad 180$

Höhne, L.; Schlotter, C. M.; Reiz, K.: Häufigkeit der Keimbesiedlung des Neugeborenen bei grünem Fruchtwasser und/oder Blasensprung - Geburtsintervall über 12 Stunden 182 Voigt, H. J.; Lang, N.: Bedeutung der Latenzzeit nach vorzeitigem Blasensprung bei kleinen Frühgeborenen (500-1500 g) 184

Homm, C; Kainer, F.; Segerer, H.: Untersuchung über pränatale Vorgehensweise und postnatales Befinden von Kindern unter $1500 \mathrm{~g}$ Geburtsgewicht bei vorzeitigem Blasensprung 186

Wischnik, A.; Hettenbach, A.; Schmidt, R.; Zieger, W.; Hug, G.; Melchert, F.: Zum Einfluß von Magnesiumsulfat auf die Volumenbilanz bei Tokolyse mit dem Betamimetikum Fenoterol 188

Spitzer, D.; Haidbauer, R.; Staudach, A.: Langzeittokolyse bei vorzeitigem Blasensprung (VBLS) und Frühgeburt 191 Mutke, H. G.: 1st der Blasensprung der Zeitgeberfür die Geburt? 195 
Häusler, M.; Hofmann, H. M. H.; Wirnsberger, G.; Leitner, G.; Gell, G.:

Untersuchung der Abortusfrequenz in der Steiermark in den Jahren 1985-87

(Tschernobyl 26. 4. 1986) 197

Heinrich, J.: Der vorzeitige Blasensprung (VBLS) - Erfordern neonatale Reife

und Infektionsrisiko eine Alternativentscheidung oder einen Kompromiß . . 200

4. Hauptthema: Operative Therapie des Mammakarzinoms

Hauptreferat

Kubista, E.: Ablative versus konservative Therapie des Mammakarzinoms 205

Koreferat

Dadak, Ch.: Erfahrung mit der Quadrantenresektion beim Mammakarzinom.

10 Jahresergebnisse an der II. Universitäts-Frauenklinik Wien 211

Mitteilungen zum 4. Hauptthema

Lang, N.; Tulusan, A. H.; Reitzenstein, M.: Chirurgische Aspekte der bruster-

haltenden Therapie des Mammakarzinoms - Segmentresektion mit simulta-

ner Brustrekonstruktion 219

Caffier, H.; Rotte, K.; Wulf, K.-H.: Brusterhaltende Therapie des Mamma-

Carcinoms, bisherige Erfahrungen mit der Würzburger Methode bei $141 \mathrm{~Pa}-$

tientinnen 223

Schönegg, W. D.; Keppke, E. M.; Pinotti, J. A.; Schäfer, A., Schmidt-Goll-

witzer, K.: Brusterhaltende Krebschirurgie unter plastisch-rekonstruktiven

Gesichtspunkten 226

Inhalt

$\mathrm{X}$

Göretzlehner, G.; Machill, G.; Grimm, U.; Knoll, W.; Seidlitz, G.; Grabow, D.:

Genetische, biochemische und genomische pränatale Diagnostik bei genetischen Defekten 278

Flores-Genger, Heidrun; Husslein, P.: Chorion-Biopsie 280

Concin, H.; Berlinger, A.; Bösch, H.; Sperl, A.; Sader, E.; Voller, Ch.;

Rosenkranz, W.: Erfahrungen mit der transabdominalen Chorionzotten-

biopsie 282

Tews, G.; Arzt, W.; Hintermüller, P.: Pränatale Diagnostik nach teratogenen

Einflüssen 283

Feiks, A.; Rudelstorfer, R.; Deutinger, J.; Chalubinski, Kinga; Nowotny, Christine; Gruber, W.:

Periphere Rheographie versus Doppler-Flow-Messung: konkurrierende Verfahren zur

Früherkennung der Plazentainsuffizienz? . . . 288

Endler, Margit; Nowotny, Christine; Balcke, P.; Derfler, K.: Proteinurie in der

Schwangerschaft - ein prognostischer Parameter 290

Oberlechner, E.; Seidel, H.; Stechele, F.; Högel, B.; Fichtel, G.; Schramm, Th.;

Rehder, H.; Rothemund, E.: Pränatale Diagnose einer familiären Akinesie-

Hypolinesie-Sequenz (Pena Shokeir Phänotyp) 292

Freie Vorträge: Endokrinologie

Husslein, P.: Die Behandlung der ,stehenden” Tubargravidität mit Prostaglandinen 294

Kirchebner, P.; Lechner, W.; Daxenbichler, G.; Marth, Ch.: Steigerung der

spontanen uterinen Aktivität durch das Antigestagen ZK 98.299 299

Freude, G.; Leodolter, S.: Der aktuelle Stellenwert von mikrochirurgischen 
Sterilitätsoperationen bei der Behandlung der tubaren Sterilität $\quad 300$ Hönigl, W.; Urdl, W.; Winter, R.; Pusch, H.; Kessler, H.; Mayer, H. O.: Extrauteringravidität nach In-Vitro-Fertilisierung und Embryotransfer (IVF-ET) $\quad 303$

Fiedler, K.; Krüsmann, G.; Rothenaicher, M.; Hirsch, P.; Würfel, W.: 295 geborene Kinder nach IVF/ET. Eine Analyse von Schwangerschafts- und Geburtsverlauf 306

Göretzlehner, G.; Leminski, Andra; Lembke, J.; Wodrig, W., Weber, A.: Der doppelte Gn-RH-TRH-Test nach dem Abstillen mit Ethinylestradiolsulfonat nach Abort, Früh- und Termingeburt 309

Pechlaner, Ch.; Kunz, F.; Tabarelli, M.; Solder, E.; Zwierzina, W. D.; Reitsamer, R,; Koch, R.: Ein neues Testsystem zur Bewertung des Thromboembolierisikos bei Einnahme hormonaler Kontrazeptiva 312 Enzelsberger, H.; Heytmanek, G.; Kurz, Ch.; Metka, M.: Die Wirkungen von Zigarettenrauchen auf Östrogenspiegel und Knochenmasse 315

Rossmanith, W. G.: Östradiol- und Progesteronsekretion bei relativer hypophysärer LH-Pulsationsruhe während der Mittlutealphase des Zyklus 317

Freie Vorträge: Geburtshilfe

Stiglbauer, M.; Sevelda, P.; Vavra, N.; Weninger, M.; Sterniste, W.;Wagenbichler, P.: Sectio versus vaginale Entbindung der Beckenendlage bei Primiparae 319 Inhalt

XI

Walcher, W.; Petru, E.; Tscherne, G.: Der Wandel des geburtshilflichen Risikos bei jugendlichen Erstgebärenden - eine Vergleichsstudie 321

Heidegger, H.; Krone, H. A.: Zur Frage der mütterlichen Mortalität, ihrer

Definition und Erfassung 323

Schröcksnadel, H.; Brezinka, Ch.; Mitterschiffthaler, G.: Frühzeitige Erkennung der Hämolyse beim HELLP-Syndrom als wichtiger prognostischer Faktor . . 325 Schroder, W.:

Die Bedeutung klinischer Symptome für Frühdiagnose und klinischen Verlauf des HELLP-Syndroms 327

Eichhorn, K.-H.; Sander, I.; Voigsberger, E.; Groß, W.; Seewald, H.-J.: Perinatales Management bei fetalen Ovarialzysten 329

Denison, U.; Wenzl, R.; Reichel, R.; Philipp, K.: Die Erfassung der asymptomatischen Bakteriurie im Wochenbett mittels Streifentest 331

Hintermüller, P.; Kainz, Ch.; Mursch, G.;Tews, G.: PC-unterstützte Datenerfassung und Verwaltung in der Geburtshilfe 333

Luschin-Ebengreuth, G.; Scharnagl, E.: Ein Fall von Graviditätsmakromastie . . 335 Klein, M.;

Rosen, A.; Laczkovics, A.; Rokitansky, A.; Beck, A.: Schwanger-

schaften nach Herzklappenoperationen

Scholler, J.; Grünberger, W.: Zwillingsschwangerschaft nach intrauterinem

Fruchttod eines Föten 339

Freie Vorträge: Gynäkologie

Schurz, Brigitte; Huber, J.; Eppel, W.; Wenzel, R.; Knogler, W.; Reinold, E.:

Vaginosonographie als Screening-Methode in Gynäkologie und Geburtshilfe 342

Behr, J.; Winkler, M.; Lang, N.: Funktionelle Descensusformen 344 
Enzelsberger, H.; Schatten, Ch.; Barrada, M.; Kurz, Ch.: Vergleichende Untersuchung zur Effektivität eines intravesical applizierten Lidocain-Gel VS Emeproniumbromid bei Frauen mit Urge-Inkontinenz 346

Gitsch, G.; Riss, P.; Janisch, H.: Die Metroplastik nach Tompkins bei Uterusmißbildung, Technik und Ergebnisse 348

Reichel, R.; Fitz, R.; Denison, Ursula; Genger, Heidrun; Vavra, N.: Rekombinantes Interferon alpha und/oder gamma bei der Behandlung von 45 Patientinnen mit Condylomata acuminata 350

Gerstner, G. J.; Piza, H.: Musculus rectus abdominis - Teilnekrose nach Pfannenstiel $\quad 352$

Klein, M.; Marczewski, A.; Rosen, A.; Beck, A.: Hohe CA-125-Werte bei einer Patientin mit Miliartuberkulose und Ascites 355

Freie Vorträge: Onkologie

Pickel, H.: Carboplatin/Epirubicin/Prednimustin (Sterecyt) als adjuvante Chemotherapie-Kombination beim radikal operierten Ovarialkarzinom 357

Kristen, P.; Beier, H.-J.; Kaesemann, H.; Caffier, H.: Nachweis von CA-125 im Serum und Gewebe von Patientinnen mit Ovarialneoplasien 358

Paterok, E. M.; Jäger, W.; Feistel, H.; Lang, N.; Wolf, F.: REGAJ-Rezidivdiagnostik beim Ovarialcarcinom $\quad 360$

Inhalt

XII

Barrada, M.; Pateisky, N.; Schatten, Ch.; Vavra, N.: Ovarialkarzinom nach Hysterektomie 364

Sautter, Th.; Mickan, H.: Transvaginalsonographisches Staging des Zervixkarzinoms 365

Spernol, R.; Hecher, K.; Nagele, F.; Szalay, S.: Zur Frage der Peritonealisierung nach Radikaloperation des Cervixcarcinoms 367

Grünberger, W.; Weber, K.: Zur Prophylaxe von Haematomen und Lymphozelen bei Radikaloperationen 370

Lahousen, M.; Haas, H.; Pickel, H.: Adjuvante Chemotherapie beim radikal operierten Zervixkarzinom 372

Grünsteidel, W.; Schmidt, C.; Tulusan, A. H.; Merkle, E.: Antiemetische

Therapie bei Cisplatin Chemotherapien $\quad 374$

Poster

Riss, P.; Breitenecker, G.: Dystrophie der Vulva - Nomenklatur und klinisches Bild 378

Gerstner, G. J.; Kronich, W.; Adam, D.: Ceftriaxon-Konzentrationen im Subperitonealraum nach Hysterektomien 381

Kölle, D.; Anderl, P.: Simultane intraurethrale EMG- und Druckmessung zur Abklärung funktioneller Veränderungen der Harnröhre 383

Casper, F.; Seufert, R.; Querbach, S.: Computergestütztes Urodynamiksystem: „UrodynП” 385

Berlinger, A.; Bösch, H.; Concin, H.: HELLP Syndrom am Krankenhaus der Landeshauptstadt Bregenz 386

Natho, W.; Abet, L.; Bollmann, R.; Prenzlau, P.: Indikationen zur Amniofetographie (AFG) in der pränatalen Diagnostik 388 
Wild, E.; Grünberger, W.; Maly, B.: Chlamydienscreening bei über 5000 Graviden 392

Paterok, E. M.; Tulusan, A. H.; Ronay, G.: Mammacarcinom: Entwicklungen seit $1963 \quad 393$

Fuith, L. C.; Artner-Dworzak, E.; Schröcksnadel, H.; Müller-Holzner, E.;

Daxenbichler, G.; Heim, K.: Prä- und posttherapeutische Serumkonzentrationen von MCA bei Patientinnen mit Mammakarzinomen 397

Czerwenka, K. F.; Schön, H. J.; Zeillinger, R.; Kubista, E.; Spona, J.: Zellrezeptoren und Proliferationsmarker beim Mammakarzinom 400

Wartusch, B.; Weiss, D.; Heim, K.;Holböck, E.;Müller-Holzner, E.;Fuith, L. C.:

Nachweis der Papillomavirustypen 6/11, 16/18 und 31/33/35 mittels DNA/

RNA-Hybridisierung. Erste Erfahrungen 402

Mitterschiffthaler, G.; Reitter, B.; Fuith, L. C; Reider, W.: Präoperative

Risikoerfassung bei Karzinomkranken 404

Heim, K.; Müller-Holzner, E.; Pinzger, G.; Holböck, E.; Wartusch, B.: Cervikale

intraepitheliale glanduläre Neoplasie (Adenocarcinoma in situ) der Cervix . 407 Vavra, N.;

Sevelda, P.; Barrada, M.; Kucera, H.: Das primär bestrahlte Adeno-

karzinom der Zervix uteri 409

Meier, W.; Stieber, P.; Fateh-Moghadam, A.; Eiermann, W.; Hepp, H.: SCC-

Verlauf unter Chemotherapie als Prognosekriterium beim Zervixkarzinom . 411

Inhalt

XIII

Petru, E.; Lahousen, M.; Pickel, H.; Stettner, H.: Chemotherapie und prognostische Bedeutung der Resttumorgröße beim Ovarialkarzinom 413

Kainhofer, A.; Fellinger, Irene; Bichler, A.: 1 st die quantitative HCG-Messung zur

Sicherung der Verdachtsdiagnose missed abortion unnotwendig geworden? . 414

Würfel, W.; Dittmar, A.; Albert, P.; Steck, T.; Schläfke, J.; Spingler, H.;

Hertwig, I. v.: Zum Einfluß von ACTH auf die HCG-Freisetzung der

Plazenta im III. Trimenon 416

Hertwig, I. v.; Würfel, W.; Link, U.; Herzog, U.; Steck, T.; Spingler, H.;

Schläfke, J.; Albert, P.: Zur CRF-induzierten hCG-Freisetzung der humanen Terminplazenta in einem in-vitro-Kultursystem 419

Enzelsberger, H.; Heytmanek, G.; Kurz, Ch.; Metka, M.: Veränderungen von

AT-III nach Hormontherapie bei Frauen im Klimakterium 422

Reichel, R.; Philipp, K.; Spona, J.: Längerfristiges Follow-up von 14 Endome-

triose-Patientinnen, die mit dem LHRH-Analogon Buserelin behandelt

wurden 425

Reinthaller, A.; Kirchheimer, J.; Lasnik, E.; Deutinger, J.: Intrafollikuläre

Plasminogenaktivatoren und Plasminogenaktivatorinhibitor Konzentratio-

nen in Relation zur Eizellreifung 427

Steck, T.; Albert, P.; Weppler, M.; Becker, W.; Spingler, H.; Würfel, W.;

Hertwig, I. v.: Wirkung eines Intrauterinpessars auf den passiven Sperma-

transport in Uterus und Tube zum Ovulationszeitpunkt 430

Rothenaicher, M.; Würfel, W.; Krüsmann, G.; Fiedler, K.; Hirsch, P.: Die

Hysteroskopie als Alternative zur Laparaskopie für den intratubaren Ga-

metentransfer (GIFT) 432 
Krüsmann, G.; Würfel, W.; Fiedler, K.; Rothenaicher, M.: Erste Ergebnisse mit dem intratubaren Embryotransfer (IVF/IT-ET) bei cervicalen Antikörpern gegen Spermatozoenantigene 436 Spingler, H.; Würfel W.; Steck, T.; Schläfke, J.; Hertwig, I. v.; Albert, P.: Entwicklung eines hysteroskopischen Transferoskops und erste Erfahrungen in der Anwendung zum intratubaren Embryotransfer (IVF/IT-ET) 439 Hirsch, P.; Würfel, W.; Fiedler, K.; Krüsmann, G.; Rothenaicher, M.: Die Fruchtwasserpunktion zur pränatalen Diagnostik in der IVF-Schwangerschaft - Ergebnisse 1985-1987 441 Rammer, Edith; Friedrich, F.: Einfluß des Gefäßquerschnittes auf die Dopplerflußkurven beim Feten 443

Grab, D.; Hütter, W.; Keim, Th.; Terinde, R.: Dopplersonographische Untersuchungen Drillings- und Vierlingsschwangerer 446

Hütter, W.; Grab, D.; Keckstein, G.; Keim, Th.; Terinde, R.: Dopplersonogra phische Befunde bei mütterlichem Diabetes in Abhängigkeit von der Stoffwechseleinstellung 448

Voigt, H. J.; Tovar, C.; Faschingbauer, C.: Dopplersonographie bei fetaler Hydrocephalie 450

Hecher, K.; Spernol, R.; Szalay, S.: Doppler-Flow-Kurven der fetalen A. carotis int. bei Zeichen von fetal distress. Vergleiche mit Flowkurven der Umbilicalarterien $\quad 452$

Inhalt XIV

Scholler, J.; Taschner, R.: Sonographisch erfaßte simultane intra- und extrauterine Gravidität 454

Kirsch, M.; Natho, W.; Abet, L.; Bollmann, R.; Bartho, S.: Der Humanus amorphus (Akardius) 456

Kainer, F.; Wessel, J.; Struck, E.; Jiminez, E.: Sonographische Diagnose bei Partialmole mit erhöhtem HCG beim Triploidie-Syndrom 460

Schurz, Brigitte, Eppel, W.; Wenzl, R.; Knogler, W.; Reinold, E.: Frühdiagnose der Tubargravidität mit der Vaginosonographie 461

Kölblinger, D.: Praenatale Diagnostik im Routineprogramm eines Stufe-II-Screenings 463

Gerstner, G. J.; Kunze, M.: Ergebnisse der Aktion „Pille light” 469

Concin, H.; Taxer, F.; Maier, R.; Sader, E.: Postmenopausales Osteoporose-

risiko 472

Autorenregister

474

Editorial

Wie seit langem bewährt, findet jedes zweite Jahr die Jahrestagung der Österreichischen Gesellschaft für Gynäkologie und Geburtshilfe zu-sammen mit der Bayerischen Gesellschaft für Geburtshilfe und Frauen-heilkunde statt. In diesem Jahr war sie in der traditionsreichen Stadt Innsbruck.

Beide Tagungspräsidenten haben das Programm gemeinsam erstellt, die Hauptthemen wurden von Referenten beider Gesellschaften von unterschiedlichen, aber sich ergänzenden Blickpunkten beleuchtet. Auch in diesem Jahr wurden besonders aktuelle Themen behandelt: die „Wer-tigkeit des vaginalen Eingriffes” ist in unserem Fach weiterhin von großer Bedeutung; die 
„HPV-Infektionen und ihr Zusammenhang mit dem Zervixkarzinom” ist ein sehr junges Forschungsgebiet, kann aber bereits sehr interessante Ergebnisse aufweisen; der Themenkreis „Vorzeitiger Blasensprung - Infektion - Tokolyse” ist aktueller denn je; die „Operati-ve Therapie des Mammakarzinoms” ist in der Gynäkologie bereits fest verankert; die „Pränatale Diagnostik" ist ein Gebiet in dem noch sehr viele neue Erkenntnisse zu erwarten sein werden. Eine Vielzahl von Vorträgen zu den Themenkreisen „Endokrinolo-gie”, „Gynäkologie”, „Onkologie” und „Geburtshilfe” sind nahezu flä-chendeckend für unseren ganzen Fachbereich. Auch in diesem Jahr war es außerordentlich erfreulich zu bemerken, daß die Autoren offenbar selber großes Interesse an der Publikation ihrer Beiträge haben: der weitaus größte Teil der Manuskripte ist noch wäh-rend der Tagung druckfertig abgegeben worden, der weitere Teil unmit-telbar danach, sodaß der Kongreß-Band bald nach der Tagung bereits an die Mitglieder zum Versand kommen kann.

In diesem Zusammenhang gilt unser besonderer Dank den Sponso-ren, denn nur mit ihrer Hilfe war dieser Kongreß-Band möglich. Mit ihrer

Editorial

XVI

Unterstützung haben sie die Verbundenheit mit unserem Fachgebiet neuerlich unter Beweis gestellt.

Unser Dank gilt aber auch den Mitarbeitern des Verlages S. Karger, Basel, sowie der Druckerei Berger \& Söhne, Horn in Niederösterreich, für die gute Zusammenarbeit.

E. Gitsch E. Reinold 